\title{
The Triangle Inequality and Its Applications in the Relative Metric Space
}

\author{
Zhanjun $\mathrm{Su}^{1}$, Sipeng $\mathrm{Li}^{1}$, Jian Shen ${ }^{2}$ \\ ${ }^{1}$ College of Mathematics and Information Science, Hebei Normal University, Shijiazhuang, China \\ ${ }^{2}$ Department of Mathematics, Texas State University-San Marcos Texas State, San Marcos, USA \\ Email:suzj888@163.com, sipengli@126.com,js48@txstate.edu
}

Received January 10, 2013; revised April 20, 2013; accepted May 16, 2013

Copyright (C) 2013 Zhanjun Su et al. This is an open access article distributed under the Creative Commons Attribution License, which permits unrestricted use, distribution, and reproduction in any medium, provided the original work is properly cited.

\begin{abstract}
Let $C$ be a plane convex body. For arbitrary points $a, b \in E^{n}$, denote by $|a b|$ the Euclidean length of the line-segment $a b$. Let $a_{1} b_{1}$ be a longest chord of $C$ parallel to the line-segment $a b$. The relative distance $d_{C}(a, b)$ between the points $a$ and $b$ is the ratio of the Euclidean distance between $a$ and $b$ to the half of the Euclidean distance between $a_{1}$ and $b_{1}$. In this note we prove the triangle inequality in $E^{2}$ with the relative metric $d_{C}(\cdot, \cdot)$, and apply this inequality to show that $6 \leq l(P) \leq 8$, where $l(P)$ is the perimeter of the convex polygon $P$ measured in the metric $d_{P}(\cdot, \cdot)$. In addition, we prove that every convex hexagon has two pairs of consecutive vertices with relative distances at least 1 .
\end{abstract}

Keywords: Relative Distance; Triangle Inequality; Hexagon

We use some definitions from [1]. For arbitrary points $a, b \in E^{n}$, denote by $a b$ the line-segment connecting the points $a$ and $b$, by $|a b|$ the Euclidean length of the line-segment $a b$, and by $\overline{a b}$ the straight line passing through the points $a$ and $b$. Let $a_{1} b_{1}$ be a longest chord of $C$ parallel to $a b$. The $C$-distance $d_{C}(a, b)$ between the points $a, b$ is defined by the ratio of $|a b|$ to

$\frac{1}{2}\left|a_{1} b_{1}\right|$. If there is no confusion about $C$, we may use the terms relative distance between $a$ and $b$. Observe that for arbitrary points $a, b \in E^{n}$ the $C$-distance between $a$ and $b$ is equal to their $\left[\frac{1}{2}(C+(-C))\right]$-distance. Thus $d_{C}(\cdot, \cdot)$ is the metric of $E^{n}$ whose unit ball is $\frac{1}{2}(C+(-C))$. We denote by $\lambda_{n}$ the relative distance between two consecutive vertices of the regular $n$-gon. It is clear that $\lambda_{3}=\lambda_{4}=2, \lambda_{5}=\sqrt{5}-1$, and

\footnotetext{
"Su's research was partially supported by National Natural Science Foundation of China (11071055) and NSF of Hebei Province (A2013205089).

Shen's research was partially supported by NSF (CNS 0835834, DMS 1005206) and Texas Higher Education Coordinating Board (ARP 003615-0039-2007)
}

$\lambda_{6}=1$. Doliwka and Lassak [1] proved that every convex pentagon has a pair of consecutive vertices with relative distance at least $\lambda_{5}$.

In this paper we first prove the triangle inequality with respect to the relative metric of a plane convex body. Then we apply this inequality to show that $6 \leq l(P) \leq 8$, where $l(P)$ is the perimeter of the convex polygon $P$ measured in the metric $d_{P}(\cdot, \cdot)$. In the last, we prove that every convex hexagon has two pairs of consecutive vertices with relative distances at least 1 .

For simplicity, if two lines $\overline{p q}$ and $\overline{r s}$ are parallel, we write $\overline{p q} \| \overline{r s}$. Denote by $x_{1} x_{2} \cdots x_{n}$ the polygon formed by the points $x_{1}, x_{2}, \cdots, x_{n}$, and by $A(P)$ the area of the polygon $P$. A chord $p q$ of $C$ is called an affine diameter if there is no longer chord parallel to $p q$ in $C$.

Lemma 1 Let $C$ be a plane convex body, and $x, y, z$ be arbitrary three points in $E^{2}$. Then the triangle inequality $d_{C}(y, z) \leq d_{C}(x, z)+d_{C}(x, y)$ holds.

Proof. By the properties of affine transformation, we may assume that the triangle $x y z$ formed by the points $x, y, z$ is a regular triangle. Let $x_{1} y_{1}, x_{2} z_{2}$, and $z_{1} y_{2}$ be the affine diameters of $C$ parallel to $x y, x z, y z$ re- 
spectively, and let $\left|x_{1} y_{1}\right|=\mu_{1},\left|x_{2} z_{2}\right|=\mu_{2},\left|z_{1} y_{2}\right|=\mu_{3}$.

Since $x y z$ is a regular triangle, by the definition of relative distance, we need to prove the following inequality.

$$
\frac{1}{\mu_{3}} \leq \frac{1}{\mu_{1}}+\frac{1}{\mu_{2}}
$$

Take the lines $\overline{x_{1} u}$ and $\overline{x_{2} v}$ through the points $x_{1}$ and $x_{2}$, respectively, such that they are parallel to $\overline{z_{1} y_{2}}$, where $u$ (resp. $v$ ) is the intersection point of the lines $\overline{x_{1} u}$ (resp. $\overline{x_{2} v}$ ) and $\overline{y_{1} z_{2}}$. Denote by $\mu$ the relative distance between the points $x_{1}$ and $u$. (See Figure 1) Since $z_{1} y_{2}$ is an affine diameter of $C$, we obtain $\mu \leq \mu_{3}$ and

$$
\frac{1}{2} \mu_{2} \mu_{3} \sin \frac{\pi}{3} \geq \frac{1}{2} \mu_{2} \mu \sin \frac{\pi}{3}=A\left(x_{1} z_{2} u x_{2}\right)
$$

The following equality is obvious.

$$
A\left(x_{1} x_{2} y_{1} z_{2}\right)=\frac{1}{2} \mu_{1} \mu_{2} \sin \frac{\pi}{3}
$$

By symmetry, we may assume without loss of generality that $\left|x_{1} u\right| \geq\left|x_{2} v\right|$. Then

$$
A\left(x_{2} y_{1} u\right) \leq A\left(x_{1} y_{1} u\right)=\frac{1}{2} \mu_{1} \mu \sin \frac{\pi}{3} \leq \frac{1}{2} \mu_{1} \mu_{3} \sin \frac{\pi}{3}
$$

By (2), (3), and (4),

$$
\begin{aligned}
& \frac{1}{2} \mu_{2} \mu_{3} \sin \frac{\pi}{3}+\frac{1}{2} \mu_{1} \mu_{3} \sin \frac{\pi}{3} \\
& \geq A\left(x_{1} z_{2} u x_{2}\right)+A\left(u y_{1} x_{2}\right) \\
& =A\left(x_{1} x_{2} y_{1} z_{2}\right)=\frac{1}{2} \mu_{1} \mu_{2} \sin \frac{\pi}{3}
\end{aligned}
$$

from which (1) holds and the proof is complete.

Let $P$ be a convex polygon. We denote by $b d(P)$ the boundary of $P$, and by $l(P)$ the perimeter of $P$ measured in the metric $d_{P}(\cdot, \cdot)$.

Proposition 2 For arbitrary convex polygon $P$, we have $6 \leq l(P) \leq 8$.

From Theorem 2 in [2] we know that for every convex polygon $P$ the perimeters of $P$ and $\frac{1}{2}(P+(-P))$ are equal in every distance space. Thus we may assume

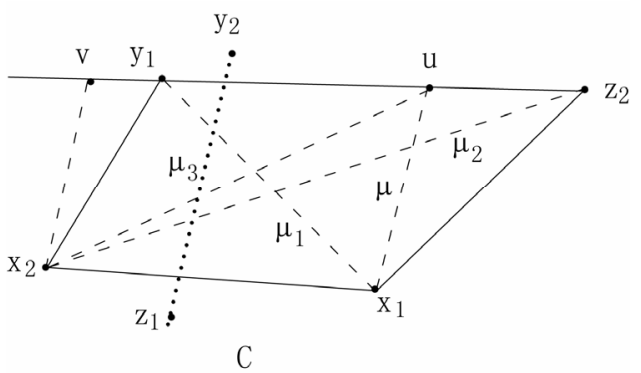

Figure 1. The figure of Lemma 1. without loss of generality that $P$ is a centrally symmetric convex polygon. We take a point $p_{1} \in b d(P)$, then there exists a point $p_{4} \in b d(P)$ such that $p_{1} p_{4}$ passes through the center of $P$. And take the points $p_{2}, p_{3} \in b d(P)$ such that $d_{P}\left(p_{2}, p_{3}\right)=\frac{1}{2} d_{P}\left(p_{1}, p_{4}\right)$ and $p_{2} p_{3} \| p_{1} p_{4}$. Then $H=p_{1} p_{2} p_{3} p_{4} p_{5} p_{6}$ is an affine regular hexagon, where $p_{5}, p_{6}$ are the antipodal points of $p_{2}, p_{3}$, respectively. It is clear that $l(H)=6$. Since the boundary of $P$ is dissected into six parts by the vertices of $H$, we consider the part between $p_{1}$ and $p_{6}$ (the other five parts can be treated similarly). Let $v_{1}, v_{2}, \cdots, v_{k}$ be the vertices of $P$ between $p_{1}$ and $p_{6}$. (See Figure 2) Draw the line-segments $p_{1} v_{1}, p_{1} v_{2}, \cdots, p_{1} v_{k}$. By Lemma 1 , we get

$$
\begin{gathered}
d_{P}\left(p_{1}, v_{k}\right)+d_{P}\left(v_{k}, p_{6}\right) \geq d_{P}\left(p_{1}, p_{6}\right), \\
d_{P}\left(p_{1}, v_{k-1}\right)+d_{P}\left(v_{k-1}, v_{k}\right) \geq d_{P}\left(p_{1}, v_{k}\right), \cdots, \\
d_{P}\left(p_{1}, v_{2}\right)+d_{P}\left(v_{2}, v_{3}\right) \geq d_{P}\left(p_{1}, v_{3}\right), \\
d_{P}\left(p_{1}, v_{1}\right)+d_{P}\left(v_{1}, v_{2}\right) \geq d_{P}\left(p_{1}, v_{2}\right) .
\end{gathered}
$$

Adding all these triangle inequalities, we obtain that

$$
\begin{aligned}
& d_{P}\left(p_{1}, v_{1}\right)+d_{P}\left(v_{1}, v_{2}\right)+d_{P}\left(v_{2}, v_{3}\right) \\
& +\cdots+d_{P}\left(v_{k}, v_{6}\right) \geq d_{P}\left(p_{1}, p_{6}\right)
\end{aligned}
$$

So we get $6=l(H) \leq l(P)$.

It is clear that we may circumscribe a parallelogram $Q:=e f g h$ about $P$ with the minimal area such that $p_{1}, p_{2}, p_{3}, p_{4} \in b d(P)$ are the midpoints of the sides ef, $f g, g h, h e$, respectively. By the properties of affine transformation we suppose without loss of generality that $Q$ is a square. Let $v_{1}, v_{2}, \cdots, v_{n}$ be the vertices of $P$ between $p_{1}$ and $p_{2}$. Let $v_{i}^{x}, 1 \leq i \leq n$, be the perpendicular projection of $v_{i}$ onto the line segment $g f$, and let $v_{i}^{y}, 1 \leq i \leq n$, be the perpendicular projection of $v_{i}$ onto the line segment ef . (See Figure 3) According to Lemma 1 , we obtain that

$$
\begin{gathered}
d_{P}\left(p_{1}, v_{1}^{y}\right)+d_{P}\left(f, v_{1}^{x}\right) \geq d_{P}\left(p_{1}, v_{1}\right), \\
d_{P}\left(v_{1}^{y}, v_{2}^{y}\right)+d_{P}\left(v_{1}^{x}, v_{2}^{x}\right) \geq d_{P}\left(v_{1}, v_{2}\right), \cdots, \\
d_{P}\left(v_{n}^{y}, f\right)+d_{P}\left(v_{n}^{x}, p_{2}\right) \geq d_{P}\left(v_{n}, p_{2}\right)
\end{gathered}
$$

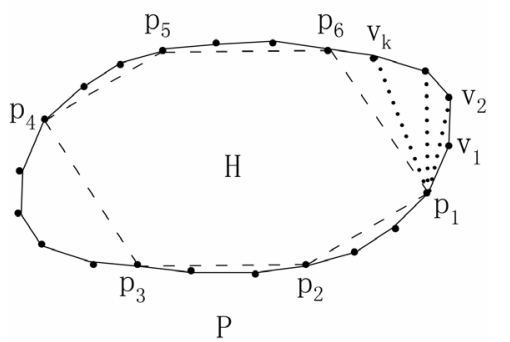

Figure 2. The figure of $6=I(H) \leq l(P)$. 


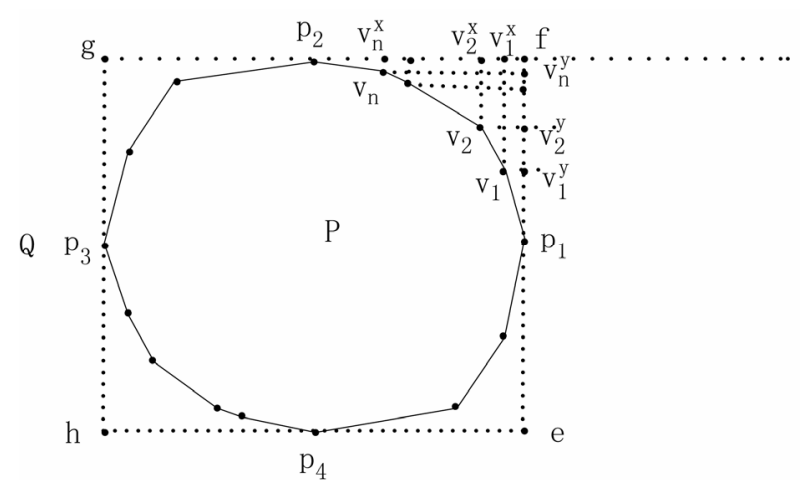

Figure 3. The figure of $l(P) \leq l(Q)=8$.

Adding all these inequalities, we have

$$
\begin{aligned}
& d_{P}\left(p_{1}, f\right)+d_{P}\left(f, p_{2}\right) \\
& \geq d_{P}\left(p_{1}, v_{1}\right)+d_{P}\left(v_{1}, v_{2}\right)+\cdots+d_{P}\left(v_{n}, p_{2}\right)
\end{aligned}
$$

Similarly, we can consider the other parts of the polygon $P$ between $p_{2}$ and $p_{3}, p_{3}$ and $p_{4}, p_{4}$ and $p_{1}$. Hence we have $l(P) \leq l(Q)=8$.

From Proposition 2 we obtain

Corollary 3 Every convex hexagon has a pair of consecutive vertices with relative distance at least 1 (that is, $\lambda_{6}$ ).

By the following Lemma [3], we give a stronger result than Corollary 3.

Lemma 4 Let $C$ be a plane convex body. We can circumscribe a parallelogram $P$ about $C$ such that the midpoints of a pair of opposite sides of $P$ belong to $C$.

Theorem 5 Every convex hexagon has two pairs of consecutive vertices with relative distances at least 1 .

Proof. Denote by $H$ the given convex hexagon. By Lemma 4, we can circumscribe a parallelogram $P$ about $H$ such that the midpoints of the opposite level sides of $P$ belong to $H$. If $H$ is a degenerate hexagon, then the result is obvious. Hence we consider the following three cases.

Case 1. The parallelogram $P$ has two sides, each of which contains exactly two vertices of $H$.

This case contains two different configurations, as shown in Figure 4. We first consider (1) in Figure 4. Since the segment $a c$ is an affine diameter of $H$, we get $d_{H}(a, c)=2$. By Lemma 1, we obtain $d_{H}(a, b)+d_{H}(b, c) \geq d_{H}(a, c)=2$. Then either $d_{H}(a, b) \geq 1$ or $d_{H}(b, c) \geq 1$. Similarly, $d f$ is an affine diameter of $H$, so either $d_{H}(d, e) \geq 1$ or $d_{H}(e, f) \geq 1$. Then consider (2) in Figure 4. Since $d$ is the midpoint of the side $y z$ of $P$, the segments $d c$ and de are not less than half of their affine diameters, respectively. Then we obtain that $d_{H}(c, d) \geq 1$ and $d_{H}(d, e) \geq 1$.

Case 2. $P$ has exactly one side which contains two vertices of $H$.

If these two vertices of $H$ belong to $x y$ or $w z$,

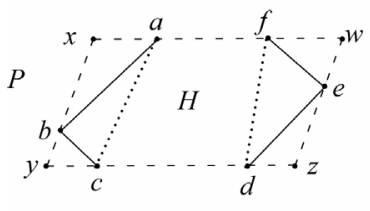

(1)

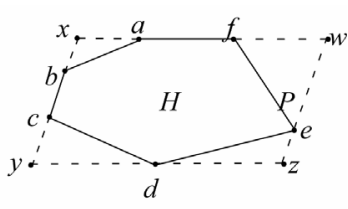

(2)
Figure 4. Case 1.

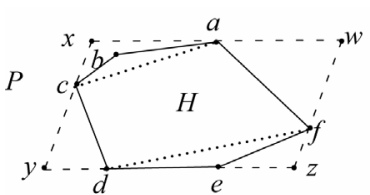

(1)

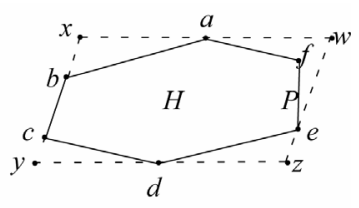

(2)
Figure 5. Case 2.

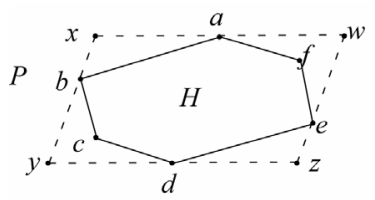

(1)

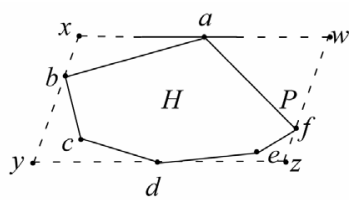

(2)
Figure 6. Case 3.

then the result is clear, see (2) in Figure 5. Otherwise, since $P$ and $H$ have five points in common, the remaining vertex of $H$ must be located inside one of the four triangular regions bounded by $P$ and $H$. See (1) in Figure 5. Since $a$ is the midpoint of the side $x w$ of $P$, we get $d_{H}(a, f) \geq 1$. Moreover, one of the segments $a c$ and $d f$ must be an affine diameter of $H$, say $d f$, then we obtain that either $d_{H}(e, d) \geq 1$ or $d_{H}(e, f) \geq 1$.

Case 3. Every side of $P$ contains exactly one vertex of $H$.

There are two different configurations in this case, as shown in Figure 6. In (1) of Figure 6, since $a$ and $d$ are midpoints of the sides $x w$ and $y z$ of $P$, respectively, we conclude that $d_{H}(a, b) \geq 1$ and $d_{H}(d, e) \geq 1$. In (2) of Figure 6, since $a$ is the midpoint of the side $x w$ of $P$, we obtain that $d_{H}(a, b) \geq 1$ and $d_{H}(a, f) \geq 1$. The proof is complete.

\section{REFERENCES}

[1] K. Doliwka and M. Lassak, "On Relatively Short and Long Sides of Convex Pentagons," Geometriae Dedicata, Vol. 56, No. 2, 1995, pp. 221-224. doi:10.1007/BF01267645

[2] I. Fáry and E. Makai Jr., "Isoperimetry in Variable Metric," Studia Scientiarum Mathematicarum Hungarica, Vol. 17, 1982, pp. 143-158.

[3] M. Lassak, "On Five Points in a Plane Body Pairwise in at Least Unit Relative Distances," Colloquia Mathematica Societatis János Bolyai, Vol. 63, North-Holland, Amsterdam, 1994, pp. 245-247. 